\section{Performance de Glicosimetro Utilizado no Automonitoramento Glicêmico de Portadores de Diabetes Mellitus Tipo I}

\section{RESUMO}

Este estudo prospectivo avaliou a dose mínima de sangue, precisão e exatidão da glicemia capilar obtidos em glicosímetro digital. Foram avaliados 108 portadores de diabetes mellitus tipo 1 (DM1), adolescentes e adultos, de ambos os sexos, recrutados junto à Clínica de Diabetes do Royal Victoria Hospital, McGill University, Canadá, durante 6 meses. No monitoramento capilar, foi utilizado glicosímetro AccuChek Compact (Roche). Para o volume, testaram-se 6 amostras de sangue, em três glicosímetros, utilizando o desenho cross-over (432 leituras). Para exatidão, comparou-se 100 amostras de sangue arterial e venoso, testadas no glicosímetro e no laboratório. Para precisão, testou-se repetidamente duas amostras de sangue venoso e soluções-controle. Os resultados demonstraram que o volume de 3,0 $\mu \mathrm{L}$ de sangue é suficiente para leitura reprodutivel. Os resultados da glicemia venosa e capilar obtidos pelo glicosímetro e testadas no laboratório não apresentaram diferença estatisticamente significativa $(p>0,05)$. Comparação dos valores de glicemia capilar medida pelo glicosímetro com glicemia venosa e capilar medida no laboratório resultou em coeficientes de correlação de 0,9819 e 0,9842, respectivamente. Estes dados confirmam a alta exatidão e precisão do glicosímetro testado. $O$ estabelecimento de punção digital de 3,0 $\mathrm{hL}$ pode ter impacto positivo na aderência ao automonitoramento. (Arq Bras Endocrinol Metab 2006;50/3:541-549)

Descritores: Diabetes mellitus; Automonitoramento da glicemia; Glicosímetros; Glicemia capilar

\begin{abstract}
Performance of Glucometer Used for Self-monitoring Blood Glycaemia in Type 1 Diabetic Patients.

This prospective study assessed the minimum volume of blood, the precision and the accuracy of capillary glycaemia obtained with the use of a digital glucometer. A total of 108 diabetic individuals were enrolled, teenagers and adults of both genders, from the Diabetes Clinic of the Royal Victoria Hospital, McGill University, Canada, in 6 months. Glycaemia monitoring was performed using an AccuCheck Compact (Roche) glucometer. For the volume, 6 samples of blood were tested, on tree glucometers, using a crossover design (432 results). For accuracy, 100 samples of venous and arterial blood, measured with the glucometer and on a clinical laboratory were compared. For precision, 2 samples of venous blood and solution controls were repeatedly tested. Results demonstrated that a volume of $3.0 \mu \mathrm{L}$ of capillary blood is sufficient for reproducible results. Measurements of venous and capillary glycaemia did not differ statistically when obtained with the glucometer or from a clinical laboratory $(p>0.05)$. Comparison of capillary glycaemia measured with the glucometer with venous and capillary glycaemia obtained from the laboratory resulted in a correlation coefficient of 0.9819 and 0.9842 , respectively. These observations confirm the accuracy and precision of the tested glucometer. The establishment of a mini-
\end{abstract}

perspectivas

\author{
Giane Sprada Mira \\ Lys Mary Bileski Candido \\ Jean François rale
}

Programa de Pós Graduação em Medicina Interna e Departamento de Nutrição (GSM, LMBC) da Universidade Federal do Paraná, Curitiba, PR, Brasil; e McGill University (JFY), Montreal, Canadá.
Recebido em 05/08/05

Revisado em 13/12/05 Aceito em 13/03/06 
mum digital punction of $3 \mu \mathrm{L}$ may have positive impact upon the compliance to auto-monitoring routines. (Arq Bras Endocrinol Metab 2006;50/3:541-549)

Keywords: Diabetes mellitus; Blood glucose monitoring system; Glucometers; Capillary glycemia

O Diabetes mellitus tipo l (DMl) é uma doença crônica, caracterizada pelo comprometimento do metabolismo da glicose, que ganhou status de epidemia mundial tornando-se hoje um importante e crescente problema de saúde pública $(1,2)$. As complicações decorrentes do controle glicêmico deficiente contribuem para redução da qualidade de vida do portador de diabetes. Assim, o tratamento moderno do DM está direcionado à prevenção das complicações crônicas pela doença, a fim de evitar maior sofrimento ao paciente e custo mais elevado com o tratamento. O tratamento multiprofissional, que inclui esquemas de insulina, monitorização capilar da glicose, contagem de carboidratos e planejamento dietético, e o envolvimento constante e harmonioso do paciente com a equipe de saúde são fundamentais para que as recomendações sejam seguidas e o tratamento seja efetivo $(3,4)$.

O automonitoramento da glicose é considerado uma ferramenta importante para o controle do DMl. Amplos estudos, como o Diabetes Control and Complications Study (DCCT) e o UK Prospective Diabetes Study (UKPDS), demonstraram o impacto benéfico do autocontrole glicêmico com redução dos riscos de retinopatias, nefropatias e neuropatias (5-7).

A Associação Canadense de Diabetes publicou, em 1998, e revisou em 2001, as diretrizes clínicas para o acompanhamento do DM que prevê, entre outras ações, teste de hemoglobina glicosilada a cada 2 a 4 meses e o automonitoramento da glicose com, no mínimo, 4 testes ao dia $(8,9)$.

No Brasil, este hábito ainda não é uma constante, sendo uma das principais causas o alto custo com o aparelho e fitas reagentes; deste modo, o tratamento preventivo ainda não é praticado fora dos grandes centros de atendimento ao portador de diabetes.

O avanço tecnológico dos glicosímetros vem permitindo a realização da glicemia capilar pelo próprio paciente no mínimo 3 vezes ao dia, sem a necessidade de recorrer ao laboratório. Porém, alguns fatores são determinantes na eficácia destes aparelhos, tais como o grau de dor, a facilidade do uso dos monitores e a fidedignidade dos resultados.
A freqüência e a constância da realização da glicemia capilar, também chamada glicemia em "ponta do dedo", é influenciada pelo desconforto causado pelo alto número de terminações nervosas presentes neste local (4). A dor constante e o aparecimento de calosidades nos dedos diminuem o número de testes realizados ao longo do tempo, prejudicando o controle metabólico do DM (10). Alguns trabalhos recentes apresentam sítios alternativos para glicemia capilar com alto grau de aceitação, porém pouco utilizados (4).

Outro fator importante é a exatidão e a precisão dos resultados oferecidos pelos glicosímetros. Velazquez e Climent (2003) avaliaram a exatidão de glicosímetros em 40 pacientes diabéticos não hospitalizados num serviço de saúde de Porto Rico. Os valores foram comparados aos obtidos por ensaio laboratorial. Testes estatísticos como análise descritiva e de correlação e teste $t$ revelaram que os resultados obtidos pelos glicosímetros foram exatos; porém, os autores alertam para o fato de que os pacientes devem receber treinamento para o correto manuseio do equipamento (7), o que é corroborado pela literatura (11).

Vários são os fatores que podem alterar os resultados obtidos nestes aparelhos, como o volume da amostra de sangue e o manuseio incorreto, tanto da fita reagente quanto do próprio glicosímetro. Muitos pacientes aprendem como utilizar o glicosímetro apenas pelas instruções do manual. Contudo, a Associação Americana de Diabetes (ADA) recomenda que o automonitoramento da glicemia, assim como outros fatores, seja parte do programa de educação ao portador de diabetes, e seja regularmente revisto para prevenção de problemas causados por resultados glicêmicos incorretos (3).

Para avaliar a fidedignidade dos dados obtidos com o uso de glicosímetros, este estudo avaliou, em uma população canadense, a exatidão e precisão dos resultados da glicemia capilar em "ponta de dedo", assim como o volume mínimo de sangue necessário para se obter um resultado confiável. Estes dados foram comparados aos obtidos através de análises em laboratório clínico.

\section{CASUÍSTICA E MÉTODOS}

Foi realizado um estudo comparativo, prospectivo, em pacientes portadores de DMl atendidos no Metabolic Day Centre do Royal Victoria Hospital (RVH) (McGill University, Canadá). Não houve restrição quanto a sexo, idade ou duração do diabetes. O único 
critério de inclusão foi a concordância do paciente em se submeter a todos os testes solicitados. O projeto foi aprovado pelo comitê de ética do Royal Victoria Hospital, e os pacientes assinaram um termo de compromisso livre e esclarecido.

No estudo foram avaliados: (i) o menor volume de sangue a ser empregado no glicosímetro para a obtenção de resultados confiáveis, e (ii) a precisão e exatidão dos resultados obtidos com o glicosímetro.

\section{População}

$\mathrm{Na}$ avaliação da exatidão foram recrutados 122 portadores de $\mathrm{DMl}$, adolescentes e adultos, por um período de seis meses consecutivos. Os critérios para inclusão no estudo foram: paciente ser portador de DMl e concordar em participar do estudo. Para 22 pacientes, o resultado de pelo menos uma amostra de sangue não estava disponível; estes pacientes foram excluídos do estudo. Todo o grupo dos 100 pacientes restantes, distribuído em 46 mulheres e 54 homens, foi testado para os parâmetros de glicemia capilar do glicosímetro (capmet), glicemia capilar do laboratório (caplab), glicemia venosa do glicosímetro (venmet) e glicemia venosa do laboratório (venlab).

Um paciente de cada sexo foi selecionado ao acaso, normoglicêmico ou não, para a avaliação da precisão.

Para o teste de volume mínimo foram analisadas amostras de sangue de 6 pacientes $(3$ mulheres e 3 homens) apresentando valores de glicemia entre 9 e 12 $\mathrm{mmol} / \mathrm{l}$ (normoglicêmicos).

\section{Glicosímetro}

O glicosímetro testado neste estudo foi o AccuChek Compact, que possui um sistema de cartucho com 17 fitas reagentes (método glicose oxidase) integrado ao aparelho, com decodificação automática, que é acionado por apenas um toque. $\mathrm{O}$ cartucho acionado libera a fita reagente na posição exata para a aplicação da gota de sangue. O resultado é fornecido em 13 segundos e, com mais um toque, a fita é descartada. De acordo com o fabricante, este mecanismo diminui o manuseio direto com a fita reagente, reduzindo o risco de erro no momento do teste. O monitor armazena até 100 registros glicêmicos com datas e horários e fornece uma média de 7 dias de resultados.

\section{Volume de sangue}

$\mathrm{Na}$ primeira etapa foram avaliadas amostras de sangue venoso de 6 pacientes portadores de DMl (normoglicêmicos) atendidos na clínica de endocrinologia do Royal Victoria Hospital. Foram excluídos pacientes que apresentaram valores glicêmicos fora dos pré-estabelecidos, entre 9 e $12 \mathrm{mmol} / \mathrm{L}$, medidos previamente pelo laboratório central. O sangue venoso foi colhido por um técnico do laboratório do Metabolic Day Centre. A amostra de sangue foi mantida no gelo até sua utilização, sendo posteriormente enviado ao McGill Nutrition Centre, onde foram aplicados os testes com o glicosímetro. Quantidades variadas deste sangue foram obtidas utilizando uma pipeta medidora com volumes específicos de $0,5,1,2,3$ e 5 microlitros, e aplicados nas fitas Softclik Select para a leitura da glicemias. Para fazer a leitura das diferentes amostras pipetadas foram utilizados três glicosímetros da marca AccuChek Compact Meter - Roche Diagnostics. Foram testados todos os volumes acima citados, tendo a quantidade de 5 microlitros como padrão, seguido de dois outros valores menores subseqüentes, aplicados simultaneamente em 3 glicosímetros diferentes e repetidos em ordem reversa, como mostra o cross-over da tabela 1. Esta seqüência foi repetida uma vez para cada paciente. As amostras que apresentaram erro na leitura foram registradas da mesma maneira. Com este modelo foram realizadas 432 leituras.

\section{Precisão}

Para se avaliar a precisão dos resultados, foram utilizados 3 glicosímetros (tabela 2) AccuChek Compact Meter Roche Diagnostics, em duas situações. O primeiro teste foi com as duas amostras das soluções-controle (Gl e G2), que acompanham o glicosímetro, as quais foram testadas 20 vezes em três diferentes glicosímetros. O segundo teste teve o mesmo procedimento, porém foram utilizadas duas amostra de sangue venoso (em qualquer estado glicêmico), de 2 diferentes pacientes portadores de DMl atendidos da Clínica de Endocrinologia durante sua consulta de rotina. As amostras de sangue foram testadas 20 vezes nos mesmos glicosímetros em que foram lidos os padrões. Este procedimento resultou em 60 leituras de glicemia para cada uma das amostras e controles testados. As sessenta leituras/amostra foram, então, utilizadas no cálculo da média, o desvio-padrão e o coeficiente de variação $(\mathrm{CV} \%)$ para estas amostras. As médias foram obtidas através de 20 repetições de cada lote de fita, totalizando 60 análises por amostra de sangue ou solução controle.

Durante toda a etapa, os códigos dos glicosímetros, solução-controle e fitas foram registrados e conferidos.

\section{Exatidão}

A terceira e última etapa deste estudo avaliou a exatidão dos resultados obtidos com o glicosímetro, comparan- 
Tabela 1. Cross-over para análise do volume de sangue.

\begin{tabular}{lccc}
\hline Glicosímetro & $\mathbf{1}$ & $\mathbf{2}$ & $\mathbf{3}$ \\
\hline Volume de sangue & & & \\
em microlitros $(\mu \mathrm{L})$ & 5,0 & 3,0 & 2,0 \\
& 2,0 & 3,0 & 5,0 \\
5,0 & 1,0 & 0,5 \\
& 0,5 & 1,0 & 5,0 \\
5,0 & 2,0 & 3,0 \\
& 3,0 & 2,0 & 5,0 \\
5,0 & 0,5 & 1,0 \\
& 1,0 & 0,5 & 5,0 \\
& 3,0 & 5,0 & 2,0 \\
& 2,0 & 5,0 & 3,0 \\
& 1,0 & 5,0 & 0,5 \\
& 0,5 & 5,0 & 1,0 \\
\hline
\end{tabular}

do-os com os resultados do laboratório. Recrutaram-se 100 indivíduos portadores de DMl (em qualquer estado glicêmico) do Metabolic Day Centre (RVH), durante suas consultas de rotina. As coletas foram realizadas por profissionais especializados, que faziam a tomada simultânea de sangue venoso e capilar (através da coleta de sangue na falange distal do dedo médio da mão direita ou teste da "picada do dedo").

Uma parte do sangue venoso foi imediatamente testada no glicosímetro AccuChek Compact, e o resultado foi registrado. Outra parte do sangue venoso foi coletada em um tubo de ensaio contendo fluoreto de sódio e enviada para o laboratório geral do RVH para análise da glicose plasmática através do método da glicose oxidase. O sangue obtido pelo teste da "picada do dedo", utilizando o lancetador SoftClix, grau três de perfuração, foi, da mesma forma, imediatamente testado no glicosímetro AccuChek Compact, e o resultado foi registrado. Deste mesmo sítio, coletou-se uma amostra adicional num tudo capilar, que foi imediatamente enviada ao laboratório geral para análise da glicemia. Esta amostra foi mantida no gelo até a sua centrifugação. Para a análise estatística, os resultados foram submetidos à análise de variância e adicionalmente avaliados através de regressão linear para calcular o coeficiente de correlação entre os métodos, empregando software Statistica (Statsoft). Valores de $\mathrm{p} \leq 0,05$ foram considerados estatisticamente significantes.

\section{RESULTADOS}

\section{Volume de sangue}

Resultados de análise de dispersão indicam que 3 microlitros de sangue é o volume mínimo necessário para avaliação correta da glicemia no glicosímetro
Tabela 2. Modelo para avaliação de precisão de glicosímetros.

\begin{tabular}{ccc}
\hline Paciente & Glicosímetro & Lote \\
\hline 1 & 1 & 1 \\
1 & 2 & 2 \\
1 & 3 & 3 \\
2 & 1 & 1 \\
2 & 2 & 2 \\
2 & 3 & 3 \\
\hline Padrão & Glicosímetro & Lote \\
\hline 1 & 1 & 1 \\
1 & 2 & 2 \\
1 & 3 & 3 \\
2 & 1 & 1 \\
2 & 2 & 2 \\
2 & 3 & 3 \\
\hline
\end{tabular}

testado (figura 1). No entanto, a menor dispersão foi obtida com 5 microlitros.

\section{Precisão}

As tabelas 3 e 4 descrevem o resultado das médias, desvios-padrão e coeficientes de variação de 60 testes realizados com duas amostras de soro e duas soluçõespadrão de glicose, respectivamente. A consistência das médias, refletida nos baixos valores de desvio-padrão e coeficiente de variação, indica que elevada precisão foi encontrada para o glicosímetro testado, tanto para as amostras de soro quanto para as soluções controle.

\section{Exatidão}

$\mathrm{Na}$ tabela 5 podem ser verificados os resultados das glicemias obtidos nas quatro situações testadas (glicemia em sangue venoso laboratório; glicemia em sangue venoso glicosímetro; glicemia em sangue capilar glicosímetro; glicemia em sangue capilar laboratório).

Não houve diferença estatisticamente significativa $(p>0,05)$, calculada pelo teste de Tukey, entre os métodos empregados e entre os valores para sangue venoso e arterial (tabela 6). A análise de variância dos efeitos do método $(\mathrm{p}=0,343155)$ e do tipo de sangue (venoso ou capilar, $\mathrm{p}=0,390777$ ) sobre as glicemias também não mostrou diferenças estatisticamente significativas entre os grupos testados.

A figura 2 apresenta gráficos obtidos por análise de regressão linear que demonstram a elevada correlação entre os resultados da glicemia medida simultaneamente em sangue venoso e capilar através do glicosímetro e de ensaio laboratorial. Os resultados da glicemia venosa obtida pelos dois métodos, glicosímetro e laboratório, não apresentaram diferença estatisticamente significativa $(\mathrm{p}>0,05)$ e o coeficiente 


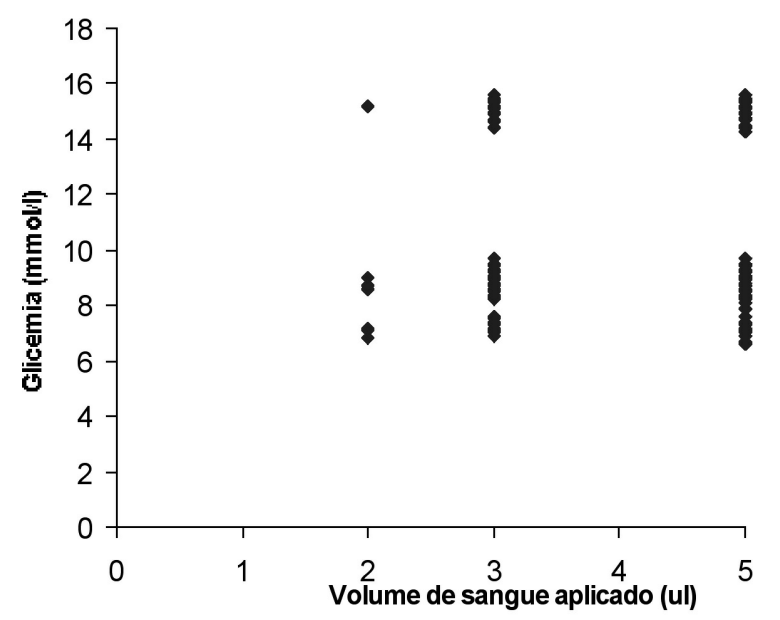

Figura 1. Glicemia obtida com diferentes volumes de sangue.

Tabela 3. Análise de precisão de duas amostras de sangue venoso.

\begin{tabular}{lcccc}
\hline Amostra & Lote de Fita & $\begin{array}{c}\text { Média } \\
(\mathbf{n}=\mathbf{2 0} ; \mathbf{m m o l} / \mathbf{l})\end{array}$ & Desvio-Padrão & CV (\%) \\
\hline Sangue \#1 & 1 & 9,98 & 0,18 & 1,80 \\
& 2 & 9,61 & 0,17 & 1,76 \\
Sangue \#2 & 3 & 10,0 & 0,17 & 1,69 \\
& 1 & 8,68 & 0,23 & 2,64 \\
& 2 & 8,61 & 0,10 & 1,16 \\
& 3 & 8,99 & 0,11 & 1,22 \\
\hline
\end{tabular}

CV \%= Coeficiente de variação.

Tabela 4. Análise de precisão das 2 soluções-controle do glicosímetro testado.

\begin{tabular}{lcccc}
\hline Amostra & Lote de Fita & $\begin{array}{c}\text { Média } \\
(\mathbf{n}=\mathbf{2 0} \text { } \mathbf{m m o l} / \mathbf{l})\end{array}$ & Desvio-Padrão & CV (\%) \\
\hline Solução \#1 & 1 & 2,46 & 0,12 & 4,87 \\
& 2 & 2,52 & 0,14 & 5,50 \\
Solução\#2 & 3 & 2,50 & 0,08 & 3,20 \\
& 1 & 9,09 & 0,53 & 5,83 \\
& 2 & 9,02 & 0,33 & 3,65 \\
& 3 & 9,06 & 0,47 & 5,18 \\
\hline
\end{tabular}

CV \%= Coeficiente de variação.

Tabela 5. Valores de glicose $(\mathrm{mmol} / \mathrm{l})$ de sangue venoso e capilar obtidos por análise laboratorial e por glicosímetros.

\begin{tabular}{lccc}
\hline Método & Média (mmol/l) & Desvio-Padrão & CV (\%) \\
\hline Sangue capilar glicosímetro & $8,89 a$ & 3,74 & 42,14 \\
Sangue capilar laboratório & $9,56 a$ & 3,76 & 39,38 \\
Sangue venoso glicosímetro & $8,88 a$ & 3,54 & 39,87 \\
Sangue venoso laboratório & $8,93 a$ & 4,08 & 45,71 \\
\hline
\end{tabular}

CV \%= Coeficiente de variação

Valores seguidos de mesmas letras não diferem estatisticamente $(p>0,05)$ 
Tabela 6. Valores de P para as variáveis da avaliação de exatidão dos glicosímetros.

\begin{tabular}{lcccc}
\hline & VENMET & VENLAB & CAPMET & CAPLAB \\
\hline VENMET & - & 0,930798 & 0,979918 & - \\
VENLAB & 0,930798 & - & - & 0,252249 \\
CAPMET & 0,979918 & $\overline{-}$ & $\overline{-}$ & 0,20766 \\
CAPLAB & - & 0,20766 & 0,20766 & - \\
\hline
\end{tabular}

As médias foram comparadas pelo teste de Tukey a $5 \%$ de significância. VENMET= glicemia em sangue venoso laboratório; VENCAP= glicemia em sangue venoso glicosímetro; CAPMET= glicemia em sangue capilar glicosímetro; CAPLAB= glicemia em sangue capilar laboratório.
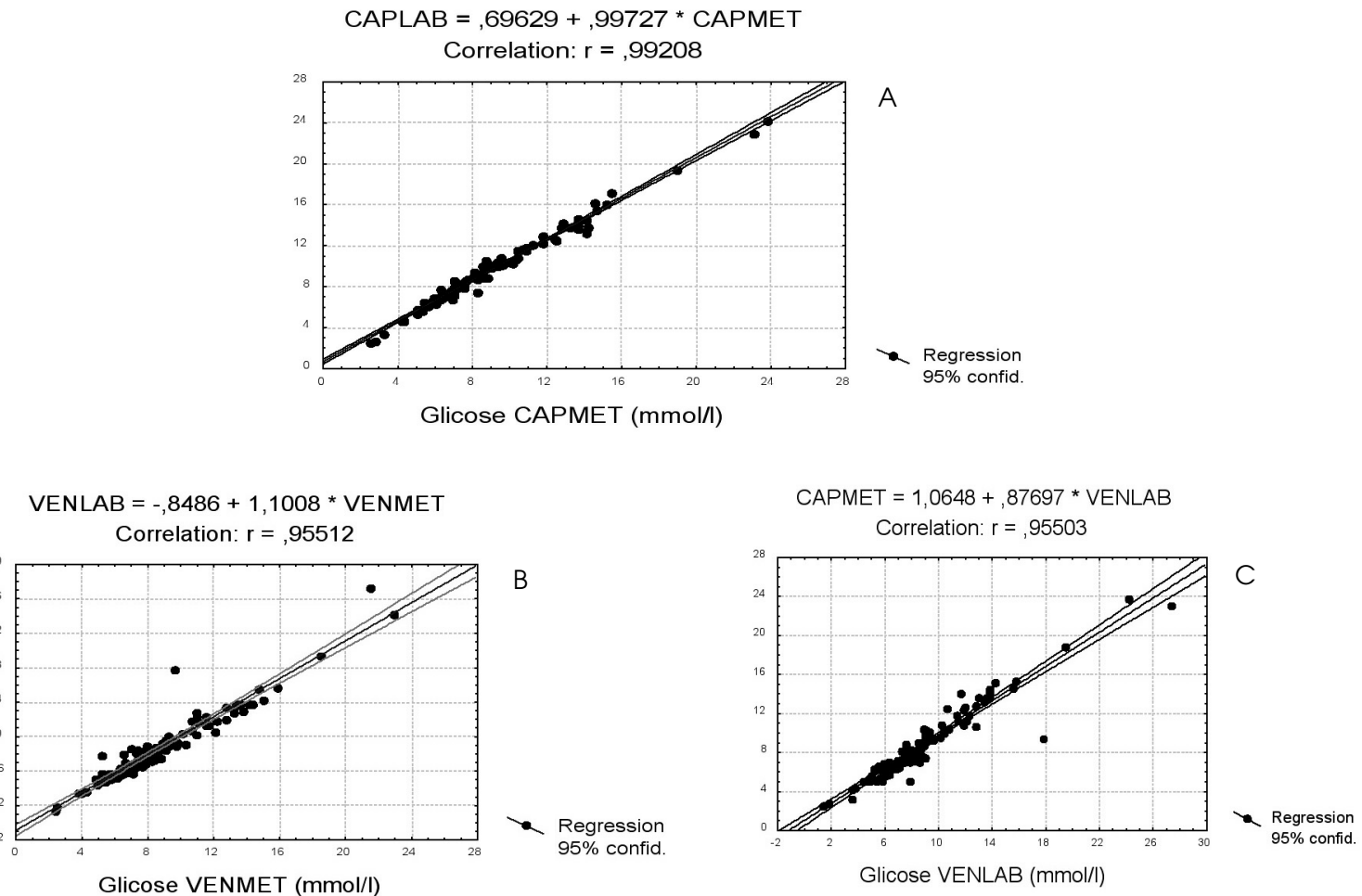

Figura 2. Correlação entre glicemia capilar e venosa obtida pelo glicosímetro e em laboratório.

$A=$ Regressão linear para valores de glicemia determinados em laboratório em sangue venoso (VENLAB) e em sangue venoso em glicosímetro (VENMET); $B$ = Regressão linear para valores de glicemia determinados em laboratório em sangue capilar (CAPLAB) e em sangue capilar em glicosímetro (CAPMET); C = Regressão linear para valores de glicemia determinados em laboratório em sangue venoso (VENLAB) e em sangue capilar em glicosímetro (CAPMET)

de correlação $\left(\mathrm{r}^{2}\right)$ foi de 0,9123 . Quando se comparou o valor da glicemia venosa medida no laboratório com a glicemia capilar medida pelo glicosímetro, também não se observou diferença significativa, e o coeficiente de correlação $\left(\mathrm{r}^{2}\right)$ foi de 0,9819 . Coeficiente ainda mais elevado foi observado quando se comparou a glicemia capilar medida no glicosímetro e no laboratório $\left(\mathrm{r}^{2}=0,9842\right)$.

\section{DISCUSSĀO}

Campanhas públicas de conscientização, associadas a métodos modernos de diagnóstico e tratamento do DM, têm resultado, ao longo das últimas décadas, tanto em aumento no número de casos diagnosticados da doença quanto em aumento da expectativa de vida dos pacientes. Estas situações levam, respectivamente, 
ao aumento dos custos com tratamento do DM e suas complicações, que têm sobrecarregado os serviços de saúde pública no mundo, e à necessidade de se garantir boa qualidade à sobrevida dos pacientes (8). Segundo a Organização Mundial de Saúde (OMS), estratégias voltadas para a prevenção de hipertensão arterial, dislipidemias e hiperglicemia associada ao DM diminuem em até $50 \%$ a incidência de internamentos por úlceras de membros inferiores ocasionadas por um pobre controle metabólico (12).

O DCCT (Diabetes Control and Complication Trial) envolveu 1.441 pacientes nos EUA e Canadá e demonstrou que, com o automonitoramento da glicemia, houve redução de $76 \%$ do risco de desenvolvimento de retinopatia primária e $54 \%$ de retardo na progressão da retinopatia. A microalbuminúria foi reduzida em $39 \%$ e a neuropatia clínica, em $60 \%$. Estes resultados são utilizados hoje como base para a indicação de um cuidadoso controle glicêmico dos pacientes portadores de diabetes, evitando ao máximo, através do automonitoramento da glicose, flutuações glicêmicas graves (6).

Assim, a prevenção de complicações associadas ao DM tem se tornado crucial no tratamento da doença. Controle glicêmico diário, contagem de carboidratos, prática de atividade física e consultas regulares ao serviço de saúde para receber treinamento e monitoramento freqüente são algumas ações consideradas, hoje, indispensáveis no acompanhamento do portador de DM.

Está bem documentado na literatura científica que a educação do portador de DMl é uma das ações mais importantes para o controle da doença e o retardo no aparecimento de suas complicações crônicas (9). Os glicosímetros são ferramentas modernas de controle de glicemia, operados pelos próprios pacientes, e com grande potencial de aplicação no auxílio da prevenção das complicações do DM. Porém, a utilização dos glicosímetros pode ser questionada em dois aspectos fundamentais: (i) a confiabilidade dos dados de glicemia obtidos, e (ii) a resistência do paciente à aderência ao procedimento, visto com freqüência como desconfortável a ponto de se tornar inviável para o uso diário. De acordo com Ferraz e cols. (2004), a dor associada ao procedimento de obtenção de sangue capilar é fator limitante para o automonitoramento da glicemia, o que predispõe a um controle metabólico deficiente e maior índice de complicações futuras (4).

Estudos anteriores já demonstraram bom potencial de utilização dos glicosímetros no monitoramento metabólico do paciente diabético, desde que sua utilização seja simples (1) e que sejam utilizados por indivíduos bem treinados $(1,11)$. Porém, a constante evolução da tecnologia aplicada na construção dos glicosímetros permite pressupor alterações nos parâmetros de qualidade destes equipamentos. Além disso, variáveis ligadas ao conforto do paciente quando da aplicação do treinamento recebido são freqüentemente negligenciadas. Vemos, portanto, como importante a atualização dos dados de confiabilidade dos glicosímetros modernos, bem como o estabelecimento de rotina de uso destes equipamentos que incentivem o paciente a aderir à sua utilização.

Neste estudo, comparou-se a qualidade dos resultados obtidos com um glicosímetro de última geração com aqueles obtidos no laboratório de análises clínicas através de métodos considerados "padrãoouro" para determinação de glicemia. Nossos dados estão de acordo com estudos anteriores que mostram boa correlação entre valores de glicemia obtidos por ambos os métodos $(1,11)$. Na verdade, enquanto a análise de performance de vários glicosímetros realizada com sangue total heparinizado detectou um coeficiente de variação de $5 \%$ para análise de precisão (1), nossos dados mostram uma precisão ainda maior, com coeficiente de variação de $1,7 \%$ (tabela 4 ).

Kendall e cols. (2005) avaliaram 100 pacientes com diabetes tipo 1 e 2 que realizavam o automonitoramento da glicemia. Os pacientes eram convidados a fazer o próprio exame com o glicosímetro. A seguir, o teste era repetido por um profissional de saúde. Ambos os resultados foram comparados a exames laboratoriais automatizados, através dos métodos da glicose oxidase e hexoquinase. Os coeficientes de correlação com os exames laboratoriais foram de 0,96 para o teste realizado pelo próprio paciente e 0,97 para o teste efetuado pelo profissional de saúde. Os autores concluíram que a educação do paciente é o elementochave para a exatidão dos resultados, uma vez que o estudo também demonstrou a exatidão e a facilidade de uso dos glicosímetros (11).

Neste estudo, detectamos elevada exatidão dos resultados de glicemia obtidos através dos glicosímetros quando comparada com os testes-padrão de glicose oxidase obtida pelo laboratório. Não foi observada diferença estatística significativa entre os dois diferentes métodos de avaliação da glicemia $(\mathrm{p}>0,05)$. Este fato está de acordo com o estudo de Kendall e cols. (2005), o qual demonstrou que esta nova geração de glicosímetros apresenta elevada exatidão, aumentando os níveis de confiabilidade dos resultados (11).

Finalmente, nossos resultados sugerem procedimentos-padrão operacionais necessários para a obtenção de resultados confiáveis de glicemia através 
do uso de glicosímetros. O estabelecimento destes procedimentos levou em conta a perspectiva do paciente portador de diabetes, e visou à diminuição do desconforto associado ao uso freqüente do equipamento.

No que se refere a fatores psicológicos que podem exercer alguma função sobre o controle da glicemia, Maia \& Araújo demonstram que grupos que apresentam baixa aceitação da doença apresentam um controle glicêmico inadequado. $\mathrm{O}$ grau de aceitação do DMl e as situações relacionadas à rotina do portador de DM têm influência direta sobre os níveis glicêmicos (13). Portanto, os dados achados neste estudo quanto ao volume da amostra de sangue a ser coletado colaboram para que se possa indicar uma punção digital menos profunda, tornando a glicemia em "ponta de dedo" melhor aceita pelo paciente.

A constatação, neste estudo, de que $3,0 \mu \mathrm{L}$ de sangue capilar são suficientes para a obtenção de resultados reprodutíveis permite punções digitais menos profundas e, conseqüentemente, menos dor associada ao procedimento. A adoção dos procedimentos aqui descritos tem potencial de reduzir a resistência do paciente à aderência ao uso do glicosímetro, sem perda da qualidade das dosagens, e com grande impacto positivo no controle das complicações do DM. Importante destacar que o teste de volume mínimo foi realizado em amostras normoglicêmicas. A extrapolação dos resultados aqui descritos para amostras hiper ou hipoglicêmicas depende de experimentos adicionais. Este critério teve sua justificativa no fato de que volumes muito baixos de sangue (abaixo dos recomendados pelo fabricante do aparelho) resultam em valores glicêmicos também baixos, o que pode prejudicar a leitura no aparelho.

É importante citar que novas tecnologias vêm sendo estudadas e utilizadas para o controle metabólico, como é o caso do CGMS (Continuous Glucose Monitoring System), um método em que é possível avaliar variações glicêmicas ao longo de $72 \mathrm{~h}$ contínuas. O CGMS é capaz de fornecer 288 leituras de glicose em 24 horas, 72 vezes mais dados que os sistemas convencionais. Um sensor subcutâneo mede a glicose intersticial a cada 10 segundos, em uma taxa que varia de $40 \mathrm{mg} / \mathrm{dl}$ a $400 \mathrm{mg} / \mathrm{dl}$, e os resultados são armazenados pela média dos valores obtidos a cada 5 minutos. Este teste apresenta alta acurácia e é utilizado principalmente na detecção de hipoglicemias e hiperglicemias não detectáveis pelos métodos convencionais, tornando a intervenção terapêutica mais eficaz e reduzindo a glico-hemoglobina (Alc) (14). Porém, o teste de monitorização contínua da glicose (CGMS) é um procedimento sugerido pelo médico a fim de se investigar um estado metabólico específico por um período máximo de três dias consecutivos. Deste modo, entendemos que, apesar do CGMS ser de extrema importância para a conduta médica perante seu paciente, a glicemia em "ponta de dedo" continua sendo o método mais utilizado no controle glicêmico diário do portador de Diabetes.

Estudos como os do DirectNet Study Group (The Diabetes Research in Children Network), de 2005, realizado com 200 crianças portadoras de DMl, onde se comparou os resultados glicêmicos oferecidos pelo método CGMS versus os resultados de 8 testes de glicemia capilar, demonstram que as médias das glicemias medidas pelos 2 métodos foram similares (15). Apesar da vantagem do número de valores glicêmicos obtidos pelo CGMS sobre a glicemia capilar, os dois métodos apresentaram resultados quase idênticos, demonstrando que ambos podem ser recomendados ao paciente, dependendo da situação a ser investigada.

Os resultados descritos neste estudo confirmam os glicosímetros modernos como ferramentas precisas e confiáveis de determinação de glicemia pelo próprio paciente, e sugerem que o estabelecimento de procedimentos-padrão de operação destes instrumentos, levando-se em consideração a perspectiva do paciente, pode minimizar o desconforto do usuário, aumentando a probabilidade de adesão ao protocolo de automonitoramento da glicemia. Em nosso entender, a combinação destes resultados proporciona uma visão mais clara dos glicosímetros como uma poderosa ferramenta de controle das complicações associadas ao DM.

\section{REFERÊNCIAS}

1. Chen ET, Nichols JH, Duh SH, Hortin G. Performance evaluation of blood glucose monitoring devices. Diabetes Technol Ther 2003;5:749-68.

2. King $H$, Aubert RE, Herman WH. Global burden of diabetes, 1995-2025: prevalence, numerical estimates, and projections. Diabetes Care 1998;21:1414-31.

3. Clement S. Guidelines for glycemic control. Clin Cornerstone $2004 ; 6: 31-9$

4. Ferraz DP, Maia FF, Araújo LR. (Fingerstick capillary glycemia versus ear lobe measurement: comparative analysis of results and patient preferences). Ara Bras Endocrinol Metab 2004;48:389-93.

5. Turner RC. The UK Prospective Diabetes Study. A review. Diabetes Care 1998;21(3):35-8.

6. The Diabetes Control and Complications Trial Research Group. The effect of intensive treatment of diabetes on 
the development and progression of long-term complications in insulin-dependent diabetes mellitus. The Diabetes Control and Complications Trial Research Group. N Engl J Med 1993;329:977-86.

7. Velazquez Medina D, Climent C. Comparison of outpatient point of care glucose testing vs venous glucose in the clinical laboratory. PR Health Sci J 2003;22:385-9.

8. Keers JC, Groen H, Sluiter WJ, Bouma J, Links TP. Cost and benefits of a multidisciplinary intensive diabetes education programme. J Eval Clin Pract 2005;11:293303.

9. Meltzer S, Leiter L, Daneman D, Gerstein HC, Lau D, Ludwig S, et al. 1998 clinical practice guidelines for the management of diabetes in Canada. Canadian Diabetes Association. CMAJ 1998;159(suppl. 8):S1-29.

10. Garg SK, Schwartz S, Edelman SV. Improved glucose excursions using an implantable real-time continuous glucose sensor in adults with type 1 diabetes. Diabetes Care 2004; $27: 734-8$

11. Kendall DM, Kaplan RA, Paulson CF, Parkes JL, Tideman AM. Accuracy and utility of a 10-test disk blood glucose meter. Diabetes Res Clin Pract 2005;67:29-35.
12. WHO. Diabetes Mellitus: the cost of diabetes. Disponivel em: http://www.who/int/en. Acessado em 07/2005.

13. Maia FF, Araújo LR. Aspectos psicológicos e controle glicêmico de um grupo de pacientes com diabetes mellitus tipo 1 em Minas Gerais. Ara Bras Endocrinol Metab 2004;48:261-6.

14. Maia FF, Araújo LR. Acurácia, efeitos na terapia insulínica e controle glicêmico e complicações do sistema de monitorização contínua da glicose em pacientes com diabetes mellitus tipo 1. Arq Bras Endocrinol Metab 2005; $49: 563-8$

15. The Diabetes Research in Children Network (DirecNet) Study Group. Eight-point glucose testing versus the continuous glucose monitoring system in evaluation of glycemic control in type 1 diabetes. J Clin Endocrinol Metab 2005:90(6):3387-91.

\section{Endereço para correspondência:}

Giane Sprada Mira

Rua Prof. Lothário Meissner 632

80210-170 Curitiba, PR.

E-mail: gianemira@ufpr.br 